\title{
Calculation of HOMs in TESLA-Cavities Using the Coupled S-Parameter Calculation Method
}

\author{
K.Rothemund*, H.-W. Glock, U.van Rienen, \\ Institut für Allgemeine Elektrotechnik, Universität Rostock, Germany
}

\begin{abstract}
The calculation of electromagnetic fields in accelerating structures is normally done by dedicated numerical solvers such as MAFIA [1]. Even if components like cavities are of cylindrical symmetry, full 3D modelling is required in order to consider the effects of power- or HOM-couplers. This implies a numerical effort significantly higher than the separate treatment of parts with and without rotational symmetry. Therefore we have developed a method called Coupled S-Parameter Calculation (CSC) which is based on a scattering parameter description. It uses S-parameters of the components found with field solving codes utilising any component's symmetry or repetition of subsections. In the paper we present a parameter variations within a TESLA9-cell-cavity with couplers and in a 4-cavity-chain in order to demonstrate the capabilities of CSC.
\end{abstract}

\section{INTRODUCTION}

Calculation of electromagnetic fields in accelerating structures of rotational symmetry can be done using $2 \mathrm{D}$ solvers. But if more complex substructures such as Higher Order Mode (HOM) couplers are attached, the symmetry is broken and full 3D modelling is necessary. This increases the numerical effort and the need of computational resources of field calculating codes rises to an extremely high level. Therefore a method called Coupled S-Parameter Calculation (CSC) has been developed which allows to split the entire structure into several subsections. The method benefits both from symmetry and repetitions of certain components, if only a small part of the geometry changes or if scattering properties can be calculated analytically. The broadband scattering-parameters (briefly: S-parameters) of the subsections are computed using ordinary field solving codes such as MAFIA, Microwave Studio ${ }^{\text {TM }}$ [1] and HFSS [2] e.g., treated as black box solvers by CSC.

\section{THEORY}

Signal reflection and transmission between the $n$ ports of an rf-component can be described by S-parameters, commonly represented by an $(n \times n)$-matrix $\mathbf{S}$ where entry $S_{i j}$ describes transmission of a signal from port $j$ to port $i$. In general it is a complex function of the frequency containing information about phase and amplitude. With $\vec{a}_{k}$ and $\vec{b}_{k}$ describing the input and output signals, the matrix $\mathbf{S}_{k}$ of the $k$-th object forms the relation $\vec{b}_{k}=\mathbf{S}_{k}(\omega) \vec{a}_{k}$.

\footnotetext{
* work supported by DESY
}

The $\mathbf{S}_{k}$ of all subsections are arranged in a block diagonal matrix $\mathbf{S}$ combining the vector $\vec{a}$ of all incident signals with vector $\vec{b}$ of all scattered signals [3]:

$$
\vec{b}=\mathbf{S} \vec{a}=\left(\begin{array}{ccc}
\mathbf{S}_{1} & & \mathbf{0} \\
& \ddots & \\
\mathbf{0} & & \mathbf{S}_{\mathbf{N}}
\end{array}\right)\left(\begin{array}{c}
\vec{a}_{1} \\
\vdots \\
\vec{a}_{N}
\end{array}\right)=\left(\begin{array}{c}
\vec{b}_{1} \\
\vdots \\
\vec{b}_{N}
\end{array}\right)
$$

All incident signals are collected in a vector $\vec{a}_{\text {inc }}$ whereas those signals travelling from one section into a neighbouring one are grouped together in the coupling vector $\vec{a}_{c o p}$. This reordering is done by a permutation matrix $\mathbf{P}$ :

$$
\vec{a}=\mathbf{P}\left(\begin{array}{c}
\vec{a}_{c o p} \\
\vec{a}_{i n c}
\end{array}\right)
$$

The feedback in the system, namely the fact that the outgoing signals of one port are incident signals at another port, is described by a feedback matrix $\mathbf{F}$; signals leaving the system are kept untouched. The inverse permutation ensures the same order of the scattered signals as in (2):

$$
\left(\begin{array}{c}
\vec{a}_{c o p} \\
\vec{a}_{s c t}
\end{array}\right)=\mathbf{P}^{-1} \mathbf{F} \vec{b}
$$

Combining (1), (2) and (3) results in the following matrix vector equation:

$$
\left(\begin{array}{c}
\vec{a}_{c o p} \\
\vec{a}_{s c t}
\end{array}\right)=\underbrace{\mathbf{P}^{-1} \mathbf{F} \mathbf{S P}}_{\mathbf{G}}\left(\begin{array}{l}
\vec{a}_{c o p} \\
\vec{a}_{i n c}
\end{array}\right),
$$

where $\mathbf{G}=\mathbf{P}^{-1} \mathbf{F} \mathbf{S} \mathbf{P}$ describes the structure of the whole system. $\mathbf{G}$ can be split into four block matrices $\mathbf{G}_{i j}$ according to the dimensions of $\vec{a}_{c o p}, \vec{a}_{i n c}$ and $\vec{a}_{s c t}$. Thus, (4)

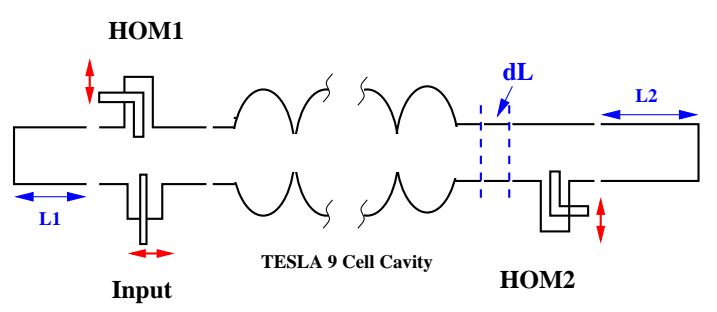

Figure 1: Sketch of the simulated structure: a combined Input-HOM-coupler section, a 9-cell-cavity and a HOMcoupler. The beam pipe is terminated by two moveable shorts to establish variable reactive loads at the beam pipe ports. 
can be written as the following system of matrix-vectorequations:

$$
\begin{aligned}
\vec{a}_{c o p} & =\mathbf{G}_{11} \vec{a}_{c o p}+\mathbf{G}_{12} \vec{a}_{i n c}, \\
\vec{a}_{s c t} & =\mathbf{G}_{21} \vec{a}_{c o p}+\mathbf{G}_{22} \vec{a}_{i n c} .
\end{aligned}
$$

The coupling between the signals is then given by $\vec{a}_{s c t}=$ $\left(\mathbf{G}_{21}\left(\mathbf{1}-\mathbf{G}_{11}\right)^{-1} \mathbf{G}_{12}+\mathbf{G}_{22}\right) \vec{a}_{i n c}$. Because $\vec{a}_{i n c}$ and $\vec{a}_{s c t}$ represent the incident and reflected waves at the external ports

$$
\mathbf{S}^{(T O T)}=\mathbf{G}_{21}\left(\mathbf{1}-\mathbf{G}_{11}\right)^{-1} \mathbf{G}_{12}+\mathbf{G}_{22}
$$

denotes the overall S-matrix $\mathbf{S}^{(T O T)}$ of the entire structure.

If all external ports are closed the structure becomes a resonator and only the coupling between the internal ports remains: $\operatorname{dim}\left(\vec{a}_{r}\right)=\operatorname{dim}\left(\vec{a}_{i}\right)=0$. The block matrices $\mathbf{G}_{12}, \mathbf{G}_{21}$ and $\mathbf{G}_{22}$ vanish and (5) reduces to

$$
\left(\mathbf{1}-\mathbf{G}_{11}\left(\omega_{k}\right)\right) \vec{a}_{c o p}^{(k)}=\mathbf{0} .
$$

Eq. (7) is only fulfilled for a discrete spectrum of resonant frequencies $\omega_{k}$ with corresponding amplitude distributions $\vec{a}_{k}$. Their determination is explained in detail in [4].

\section{APPLICATION}

In order to demonstrate the advantages of CSC, we used it to estimate the transmission between the HOM-couplers of a cavity in the accelerating channel of the TESLA Test Facility [5] (TTF). It is a chain assembled of a combined Input-HOM-coupler followed by an accelerating cavity and a single HOM-coupler rotated by $115^{\circ}$ with respect to the first HOM-coupler [6].

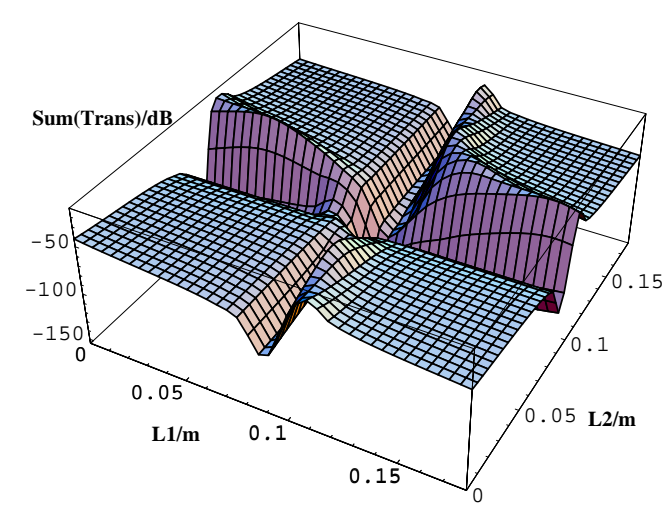

Figure 2: Summerised mutual transmissions at 2.50 GHz.

Because of its rotational symmetry the cavity is modelled in 2D polar coordinates using 110,187 nodes; Sparameter calculation was performed by MAFIA's time domain solver T2. Even in two dimensions calculation took $\approx 50 \mathrm{~h}^{1}$, caused by the structure's resonant behaviour and

\footnotetext{
${ }^{1}$ on a SUN ULTRA 2 with 2 GByte RAM and $300 \mathrm{MHz}$
}

the need of tiny time steps, which are a consequence of a special grid, prepared to achieve optimal shape approximation [7]. The HOM-couplers had to be modelled in full 3D. This was done in Microwave Studio ${ }^{\mathrm{TM}}$ which results in 225,675 and 426,216 nodes, resp. The computation of the complete set of scattering parameters $(\mathrm{a} 5 \times 5$ - and a $6 \times 6$ matrix) was performed in around two days on a $500 \mathrm{MHz}$ $\mathrm{PC}$ for each coupler section.
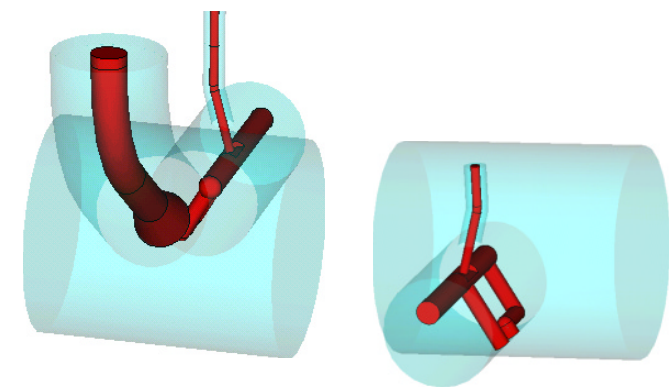

Figure 3: The DESY-type HOM-coupler (on the left with the additionally attached input coupler). These geometries are discretized in Microwave Studio ${ }^{\mathrm{TM}}$ with 426,216 and 225,675 nodes.

The entire S-matrix of the arrangement shown in Fig. 1 (with $d L$ kept 0 ) was calculated for an arbitrarily chosen frequency of $2.50 \mathrm{GHz}$ and $20 \times 20$ equally distributed lengths of $L 1$ and $L 2$. This took $32 \mathrm{~s}$ on a $500 \mathrm{MHz}$ PC. In order to identify constellations of weak external coupling the sum of the mutual transmissions (dB-scaled) was plotted vs. $L 1, L 2$ (Fig. 2). There a distinct transmission minimum appears, demonstrating the possibility of very weak coupling of the couplers.

Then the transmission between the HOM-couplers was computed by CSC as a function of frequency, which showed a strong dependence on the additional length $d L$ (see Fig. 4).

This result suggested that a similar behaviour could be

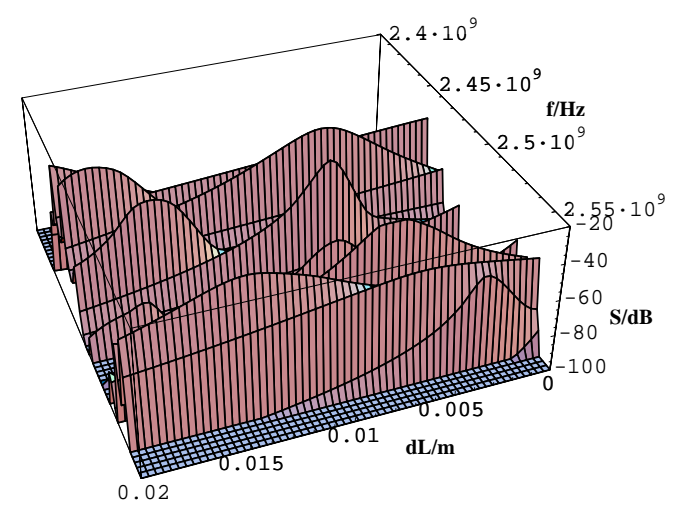

Figure 4: Coupling between the two HOM-couplers as a function of the frequency and the length $d L$. 


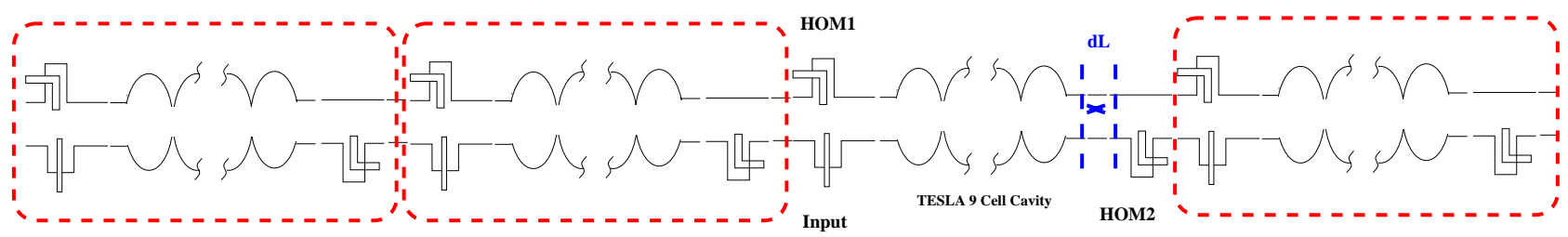

Figure 5: Chain of four TESLA-cavities with attached HOM- and Input-couplers. Between cavity 3 and HOM-coupler HOM2 extra space of variable length $d L$ is inserted to "tune" the transmission between the HOM-couplers.

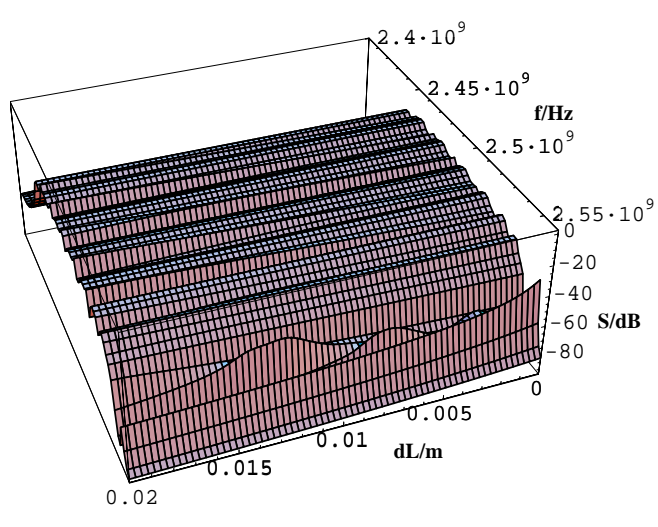

Figure 6: Coupling between couplers HOM1 and HOM1 as function of frequency and length $d L$, for most frequencies weakly varying with length.

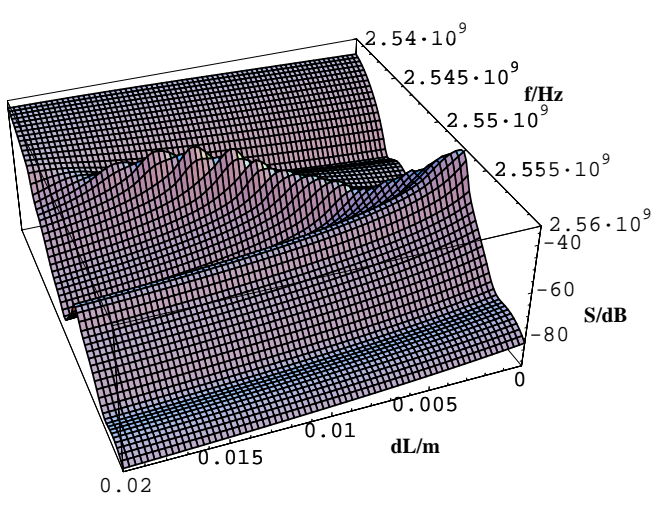

Figure 7: Detail of the plot in Fig. 6 showing a strong dependency of $d L$ resulting in transmission variation of up to $40 \mathrm{~dB}$; frequency range of $2.54 \ldots 2.56 \mathrm{GHz}$.

seen if the cavity section is build into the TESLA accelerating channel. So, in a second step the shorts were replaced by a chain of TESLA-cavities plus HOM-couplers (Fig. 5). The resulting structure consists of 4 cavity sections which requires a partitioning into 16 subsections; the dimension of the resulting scattering matrix is $16 \times 16$.

Different from the setup with shorted beam pipes then the coupling between the two HOM-couplers depends only weakly on the additional length $d L$ (Fig. 6), except for fre- quencies at the upper passband's edge (around 2.55 GHz): their resonance effects cause a change in transmission of $40 \mathrm{~dB}$, which may not be negligible.

\section{CONCLUSION}

CSC allows to calculate scattering properties of long and complex structures. It can take a lot of profit out of modularised components, especially if only a small section is intended to be varied. This was demonstrated by the calculation of the scattering properties of a TESLA-9-cell-cavity assembled with all couplers, which is not manageable in direct solvers with todays computational resources. CSC gives the additional opportunity of a fast variation of geometrical parameters over a wide range. It is shown in the paper, that CSC can manage large structures such as a 4-cavity structure fully equipped with HOM- and Inputcouplers with a reasonable computational effort, taking big advantage out of the modularised assemble.

\section{ACKNOWLEDGEMENT}

The authors would like to thank M. Dohlus for helpful hints concerning the HOM-coupler geometries.

\section{REFERENCES}

[1] MAFIA V4.24 and Microwave Studio ${ }^{T M}$ V3.0, CST GmbH, Büdinger Straße 2a, D-64289 Darmstadt, Germany.

[2] HP HFSS, Agilent Technologies, Palo Alto, USA.

[3] T.-A. Abele, Über die Streumatrix allgemein zusammengeschalteter Mehrpole, Archiv der Elektrischen Übertragung, Band 14, pp 262-268, 1960.

[4] K. Rothemund, H.-W. Glock, M. Borecky, U. van Rienen, Eigenmode Calculation in Long and Complex RF-Structures Using the Coupled S-Parameter Calculation Technique, Proceedings of the ICAP 2000, and TESLA-Report 2000-33

[5] B. Aune et. al., Superconducting TESLA cavities, Physical Reviews Special Topics - Accelerators and Beams, volume 3, pp. 1-25, (2000).

[6] R.Brinkmann, K. Flöttmann, J.Rossbach, P. Schmüser, N. Walker and H. Weise (eds.), TESLA Technical Design Report, DESY, Hamburg, March 2001

[7] H.-W. Glock, D. Hecht, U. van Rienen, Field Flatness and Non-Stationary Behaviour of the 4*7-Cell-TESLASuperstructure, Proceedings of the ICAP98, 1998. 